OPEN ACCESS

Edited by:

Baolin Guo,

Xi'an Jiaotong University, China

Reviewed by:

Yaobin Wu,

Southern Medical University, China

Xiaowen Wang,

Hong Kong Polytechnic University,

Hong Kong

${ }^{*}$ Correspondence:

Lin Tan

tanlinou@scu.edu.cn

Specialty section

This article was submitted to

Polymer Chemistry,

a section of the journal

Frontiers in Chemistry

Received: 06 July 2018 Accepted: 26 September 2018

Published: 16 October 2018

Citation:

Yin X, Wen Y, Li Y, LiU P, Li Z, Shi Y, Lan J, Guo R and Tan L (2018) Facile Fabrication of Sandwich Structural Membrane With a Hydrogel

Nanofibrous Mat as Inner Layer for Wound Dressing Application.

Front. Chem. 6:490

doi: 10.3389/fchem.2018.00490

\section{Facile Fabrication of Sandwich Structural Membrane With a Hydrogel Nanofibrous Mat as Inner Layer for Wound Dressing Application}

\author{
Xueqian Yin ${ }^{1}$, Ya Wen ${ }^{1}$, Yajing $L i^{1,2}$, Pengqing $L_{i u^{3}}$, Zhongming $L i^{3}$, Yidong Shi ${ }^{1}$, \\ Jianwu Lan ${ }^{1}$, Ronghui Guo ${ }^{1}$ and Lin Tan ${ }^{1,3 *}$ \\ ${ }^{1}$ College of Light Industry, Textile and Food Engineering, Sichuan University, Chengdu, China, ${ }^{2}$ College of Architecture \& \\ Environment, Sichuan University, Chengdu, China, ${ }^{3}$ College of Polymer Science and Engineering, Sichuan University, \\ Chengdu, China
}

A common problem existing in wound dressing is to integrate the properties of against water erosion while maintaining a high water-uptake capacity. To tackle this issue, we imbedded one layer of hydrogel nanofibrous mat into two hydrophobic nanofibrous mats, thereafter, the sandwich structural membrane (SSM) was obtained. Particularly, SSM is composed of three individual nanofibrous layers which were fabricated through sequential electrospinning technology, including two polyurethane/antibacterial agent layers, and one middle gelatin/rutin layer. The obtained SSM is characterized in terms of morphology, component, mechanical, and functional performance. In addition to the satisfactory antibacterial activity against Staphylococcus aureus and Escherichia coli, and antioxidant property upon scavenging DPPH free radicals, the obtained SSM also shows a desirable thermally regulated water vapor transmission rate. More importantly, such SSM can be mechanically stable and keep its intact morphology without appearance damage while showing a high water-absorption ratio. Therefore, the prepared sandwich structural membrane with hydrogel nanofibrous mat as inner layer can be expected as a novel wound dressing.

Keywords: sandwich structure, hydrogel nanofibrous mat, antibacterial activity, antioxidant activity, wound dressing application

\section{INTRODUCTION}

Hydrogel-based wound dressings are effective materials for the treatment of chronic wound due to the excellent exudates absorption and retaining properties, which allow them to modulate the fluid balance at the site of wound bed and maintain an microenvironment close to that present in native tissue (Fan et al., 2014; Sun et al., 2017; Zhao et al., 2017). Currently, most hydrogels are synthesized in terms of film, powder, particle and bulk 3D constructs. However, some drawbacks existing in those types of hydrogels when applying them as wound dressing materials, such as low gas and liquid permeation, insufficient surface area, poor structural integration (Ravichandran et al., 2014; Chen et al., 2015; Sun et al., 2017). Recently, hydrogel nanofibrous mats which 
can combine the advantages of both nanofibers and hydrogels have received increasing attention due to their desirable gas/liquid permeability, high surface area, highly porous structure, and excellent water uptake capacity(Brunelle et al., 2017; Sun et al., 2017; Zhao et al., 2017).

Electrospinning is an efficient and versatile technique to produce diversified nanofibers, and hundreds of polymers and their composites have been successfully processed into fibrous structure through electrospinning (Lin et al., 2012; Valizadeh and Mussa, 2014; Ahmed et al., 2015). Recently, several electrospun hydrogel nanofibrous mats, e.g., poly(vinylalcohol) (Bhowmick and Koul, 2016; Oh et al., 2016), Poly-(N-vinyl-2-pyrrolidone) (Kurniawan et al., 2016), poly(acrylic acid)-silica (Wang et al., 2016), poly(N-isopropylacrylamide) (Wen et al., 2015; Yao et al., 2015) have been reported, and the obtained hydrogel nanofibrous mats were mostly proposed to be applied as wound dressing and tissue scaffold. Nevertheless, the maintenance of intact hydrogel nanofibrous structure should be further improved during the functions performing due to the intrinsic poor mechanical stability (Fan et al., 2014; Gonzalez et al., 2014; Wang et al., 2015; Wu et al., 2017), thus additional bottom and top protection barriers for hydrogel nanofibrous layer are necessary (Xia et al., 2015; Kim et al., 2017). Regarding the wound dressing application, susceptible to water erosion and bacterial infection are fatal for wound healing, the membranes possessing two hydrophobic surfaces and one inner hydrophilic layer have greatly promising prospects in wound dressing application accordingly, while each layer can have and perform its own function separately, such as antibacterial property, high water uptake capacity, water resistance against erosion (Fan et al., 2014; Xu et al., 2015; Zhao et al., 2017). In other words, sandwich structure may be an ideal candidate for wound dressing fabrication. However, the conventional electrospun nanofibrous membrane is single layer or double layers. Obviously, the former type has the same wettability for its both surfaces, and the problem of relatively low adhesive force between two individual layers exists in latter type (Tan et al., 2015c; Trinca et al., 2017). Therefore, the fabrication of sandwich structural membrane with hydrogel nanofibrous mat may address the above concerns.

Both electrospun polyurethane and gelatin are popular materials for wound dressings application (Li et al., 2016; Resmi et al., 2016; Sahraro et al., 2016; Nian et al., 2018). Polyurethane (PU) currently is one of the most widely applied synthetic polymers. Compared with conventional polymers, PU possesses comprehensive excellent performances on following aspects, including hydrophobic property, easy preparation, recyclability, and good mechanical features, such as the stretching capability and elasticity (Janik and Marzec, 2015; Kucinska-Lipka et al., 2015); Gelatin (GE) is a natural protein which is derived from the partial and irreversible hydrolysis of collagen, and it also has been extensively employed as a hydrogel polymer (Zhao et al., 2015, 2018), but the poor intrinsic mechanical property of gelatin hinders its application, and the primary way to exert the functions of gelatin is to combine with other polymers (Tan et al., 2015c; Feng et al., 2016; Trinca et al., 2017).

Herein, we put forward the concept of fabricating one novel sandwich structural membrane (SSM) consisting of two hydrophobic electrospun PU layers as bottom and top surfaces, respectively, and one hydrophilic inner layer of gelatin hydrogel nanofibrous mat. Additionally, in order to accelerate the potential wound healing process, both antibacterial activity and free radicals scavenging capacity are introduced into such SSM. We demonstrate that the facilely fabricated SSM shows good performance on antibacterial activity, antioxidant property, water vapor transmission rate, mechanical and water uptake capacity. Therefore, the fabricated SSM in this study can be highly recommended as an ideal cover dressing for promoting wound healing.

\section{MATERIALS AND METHODS}

\section{Materials}

Polyurethane and antibacterial agent N-halamine (N-chloro2,2,6,6-tetramethyl-4-piperidinyl, $\mathrm{Ca}$ ) were synthesized according to the previous reports, and the synthesis information of Ca is presented in Figure S1 (Tan et al., 2015a; Dong et al., 2017). Rutin was purchased from Shanghai Aladdin Biochemical Polytron Technologies Inc.; Gelatin (Type A) from porcine skin was purchased from Sigma Aldrich. N, N-dimethylacetamide (DMAc) and other solvents were purchased from Chengdu Kelong reagent company and used directly without further purification. Dehydrated polycaprolactone diol (PCL, Mn = $4,000 \mathrm{~g} / \mathrm{mol}$ ) was obtained from Perstop U.K. Ltd (United Kindom). 4,4-methylene bis(phenylisocyanate) (MDI), 1,4Butanediol (BDO) and dibutyltin dilaurate (DBTDL) were purchased from Sigma Aldrich (Unnithan et al., 2015).

\section{Synthesis of Polyurethane}

The applied polyurethane used for electrospinning in this study was synthesized according to our previous method. In particular, the obtained PU composed of PCL, MDI, and chain extender $\mathrm{BDO}$. The reaction to prepare prepolymer was conducted in a $1,000 \mathrm{~mL}$ conical flask equipped with a mechanical stirrer. PCL ( $\sim 492 \mathrm{~g}$ ) mixed with MDI ( $\sim 24 \mathrm{~g})$ for $2 \mathrm{~h}$ at $80^{\circ} \mathrm{C}$, and followed by the chain extension reaction with BDO $(\sim 100 \mathrm{~g})$ for another $2 \mathrm{~h}$ under same temperature. The whole isocyanate group content was excessive by $3 \mathrm{~mol} \%$, and the hard segment content is around $20 \%$. All the chemicals used in the synthesis process should be dehydrated with $4 \AA$ molecular sieves in advance (Tan et al., 2015a).

\section{Preparation of Gelatin-Rutin (GE-R) and Polyurethane-Antibacterial Agent (PU-Ca) Solution}

Firstly, $24 \mathrm{wt} \%$ gelatin solution was prepared by dissolving gelatin powder into a binary solvent consisting of water and formic acid with the mass ratio of 8:2 accordingly and the homogenous solution was obtained after stirring at room temperature for $3 \mathrm{~h}$; Thereafter, a calculated amount of rutin powder (10 wt $\%$ of gelatin) was added to the solution for another $2 \mathrm{~h}$ stirring under the same condition. Similarly, PU-Ca dissolved in DMAc was prepared under constant stirring at $80^{\circ} \mathrm{C}$ for $4 \mathrm{~h}$, the concentration of $\mathrm{PU}$ was fixed at $20 \mathrm{wt} \%$, and the ratio of $\mathrm{Ca}$ was $10 \mathrm{wt} \%$ of PU content. 


\section{Fabrication of Electrospun Sandwich Structural Membrane (SSM)}

SSM was prepared using a manual assembly electrospinning system. The bottom and top layers were fabricated by spinning PU-Ca solution, and the inner hydrogel nanofibrous layer was made from GE-R solution, two voltage gradients of around 1.0 and $1.8 \mathrm{kV} / \mathrm{cm}$ were applied for $\mathrm{PU}-\mathrm{Ca}$ solution and GE-R solution, respectively, and the feeding rate of $1 \mathrm{~mL} / \mathrm{h}$ was fixed during electrospinning process under an ambient condition (Relative humidity $=50 \pm 5 \%$, Working temperature $=22 \sim 26^{\circ} \mathrm{C}$ ). In the course of spinning process, we controlled the thickness of the electrospun membrane through feeding ratio, spinning time and the width for nanofiber collection, and the thickness was measured with the aid of a micrometer (Mitutoyo, Japan). The PU-Ca/GE-R/PU-Ca sandwich structure was constructed through sequential electrospinning. In our study, two SSMs were prepared by adjusting the thickness ratio among the three layers, particularly including 1:4:1 and 2:2:2, and we named such two SSMs in short as "SSM141" and "SSM222," respectively. In addition, the PU-Ca and GE-R nanofibrous mats were prepared as comparisons. SSM141, SSM222, and GE-R nanofibrous mats were crosslinked by saturated glutaraldehyde vapor for $15 \mathrm{~min}$ and followed by placing in a vacuum desiccator to remove residual crosslinker.
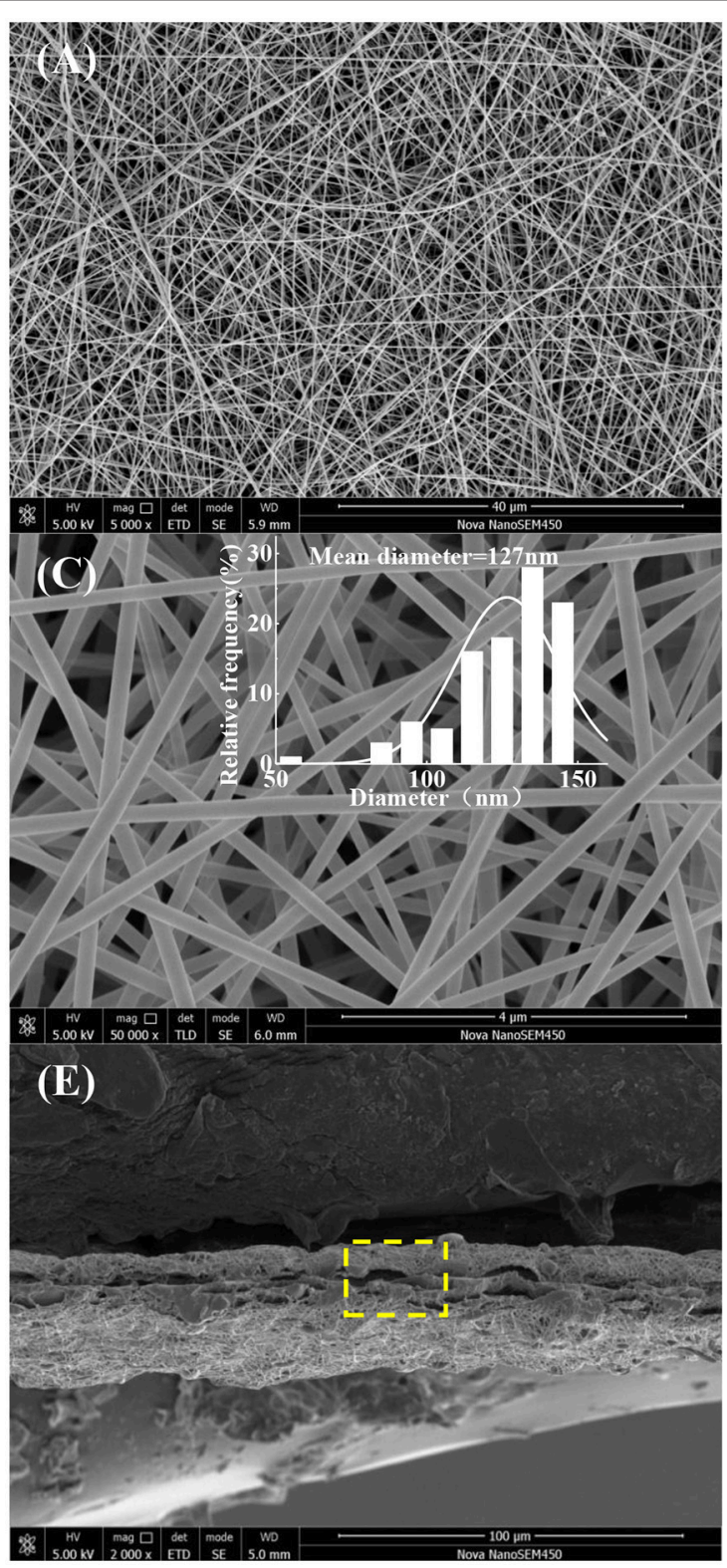
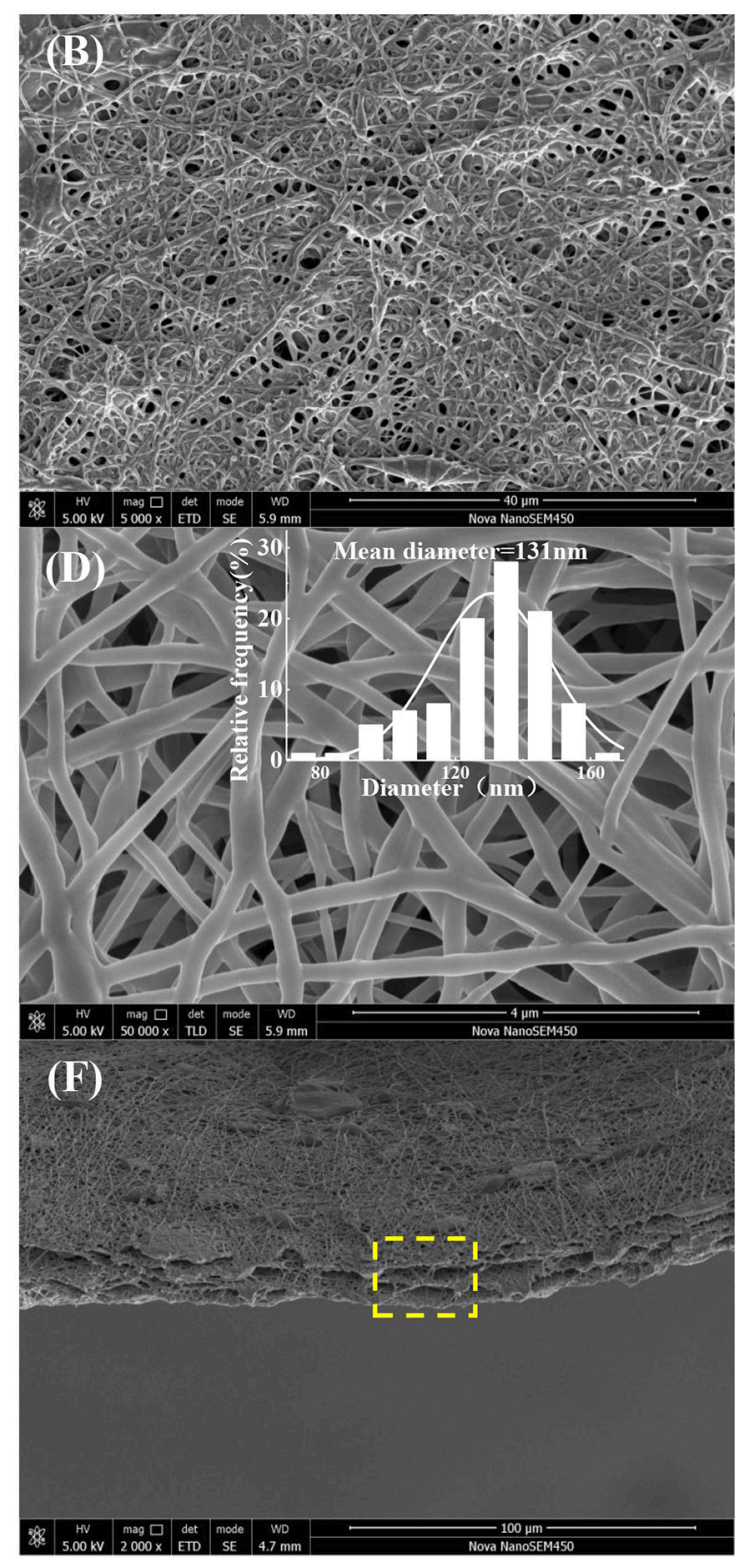

FIGURE 1 | Surface images and fibers diameter distribution (ImageJ) of GE-R (A,C), and PU-Ca (B,D), and cross-sectional images of SSM141 (E), and SSM222 (F). 


\section{Characterizations}

The surface and cross-section morphologies of nanofibrous mats were characterized by scanning electron microscope (SEM, Hitachi SU3500, Japan), and all specimens were sputter-coated

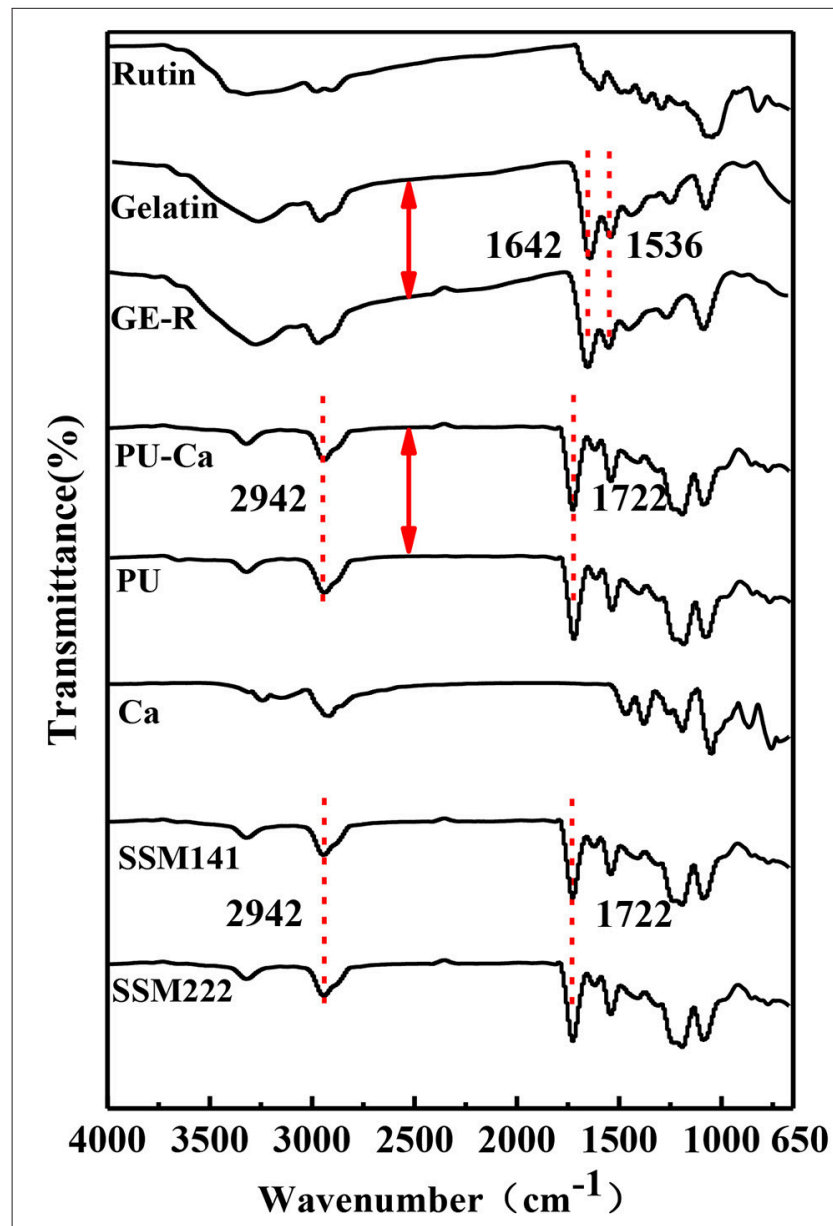

FIGURE 2 | FT-IR spectrum of each component of SSM. with gold for $20 \mathrm{~s}$ before observation. Nanofiber diameter distribution was determined by measuring the diameters of 100 randomly selected nanofibers in SEM images with the aid of ImageJ software; The chemical structure and conformation of nanofibrous mats were analyzed by attenuated total reflectance model based Fourier transform infrared (ATR-FTIR, Tracer-100, Japan) spectroscopy in the range of $650-4,000 \mathrm{~cm}^{-1}$ at room temperature; Thermal decomposition behavior was investigated by thermogravimetric analysis (DTG-60, Shimadzu, Japan) with a heating scan from 30 to $800^{\circ} \mathrm{C}$, and the flow rate of nitrogen gas was fixed at $50 \mathrm{~mL} / \mathrm{min}$; Mechanical properties was investigated using an electronic single yarn strength meter (YM061,China). The width of specimens employed was $3 \mathrm{~mm}$ and the distance between two clamps was $20 \mathrm{~mm}$, the stretching speed was 10 $\mathrm{mm} / \mathrm{min}$, and each sample was measured at least 10 times, the average values of breaking force $(\mathrm{cN})$ and elongation at break (\%) were recorded; Water contact angle (WCA) of the fabricated membranes surface was measured through a contact angle tester (Harke-SPCAX1, China) using the sessile drop method, and at least five individual values were collected and averaged.

\section{Water-Absorption Ratio and Water Vapor Transmission Rate (WVTR) Investigation}

WAR was evaluated by immersing samples in PBS buffer $(\mathrm{pH}$ $=7.4$ ) for $12 \mathrm{~h}$, and then weighted them after removing the surface water using wiping paper; WVTR was determined based on evaporation of water vapor through the testing nanofibrous mats under the temperature of 21 and $37^{\circ} \mathrm{C}$ with a continuous $\% \mathrm{RH}$, including 35,55 , and $75 \%$. WVTR was monitored by measuring the weight loss ( $\mathrm{mg}$ ) of the cups by functions of unit area $\left(\mathrm{cm}^{2}\right)$ and time $(\mathrm{h})$, the cups without samples were applied as comparisons. Tests of both WAR and WVTR were performed in triplicate.

\section{Antibacterial Activity Assessment}

Antibacterial activity of SSM against Staphylococcus aureus and Escherichia coli was investigated based on agar disk diffusion susceptibility. The samples were cut into discs with the size of $1.5^{*} 1.5 \mathrm{~cm}^{2}$, and sterilized by ultraviolet irradiation $15 \mathrm{~min}$ for
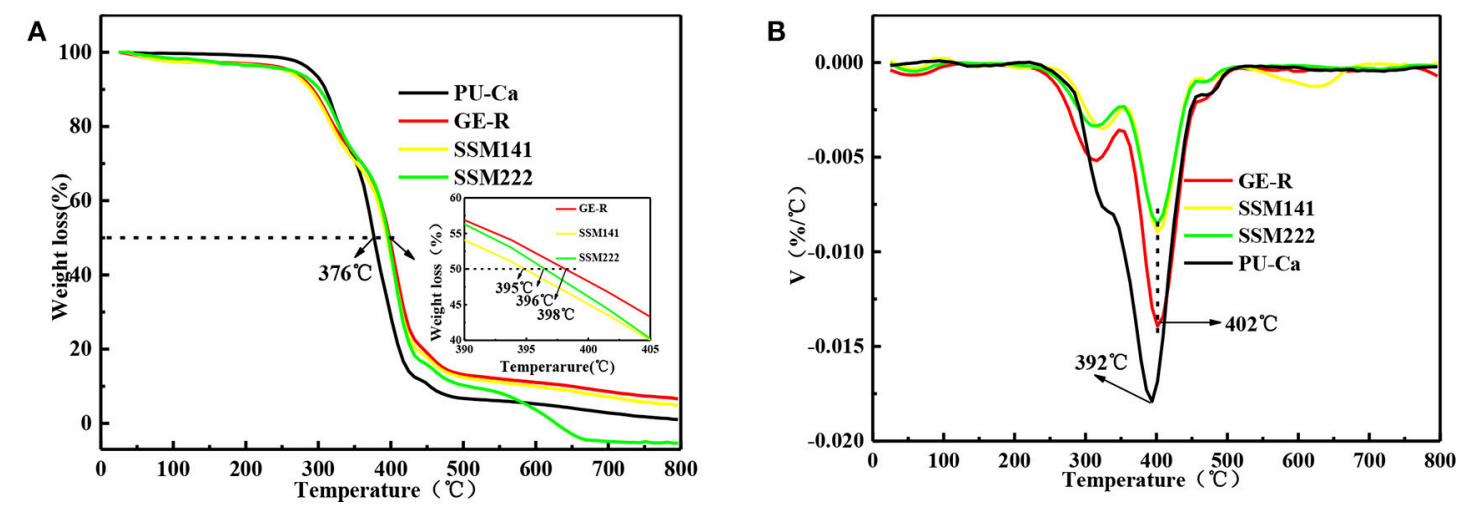

FIGURE 3 | Thermal decomposition (A) and DTG (B) curves of different electrospun membranes. 
both surfaces separately in a laminar flow hood. Gram-negative E. coli and Gram positive S. aureus were selected as representative bacteria and were cultivated overnight in an incubator, and the sterilized samples were placed on the surface of agar plates coated with the E. coli and S. aureus already diluted by PBS buffer. Subsequently, the plates were incubated at $37^{\circ} \mathrm{C}$ overnight, and then inhibition zones were observed and photographed.

\section{DPPH Radical Scavenging Activity}

The antioxidant activity of SSM were determined according to 1,1-diphenyl-2-picrylhydrazyl (DPPH) free radical-scavenging

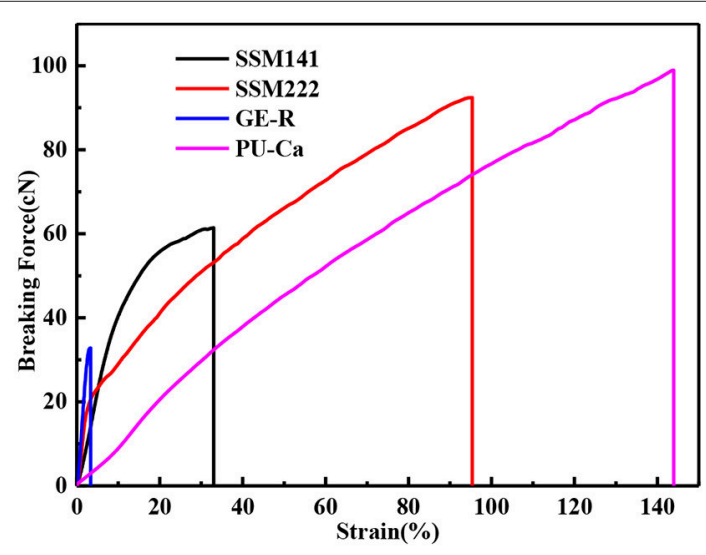

FIGURE 4 | Stress-strain curves of different membranes.

TABLE 1 | The average tensile strength, thickness, elongation at break of different membranes.

\begin{tabular}{lccc}
\hline Sample & $\begin{array}{c}\text { Membrane } \\
\text { thickness/ } \boldsymbol{\mu m}\end{array}$ & $\begin{array}{c}\text { Breaking } \\
\text { force/cN }\end{array}$ & $\begin{array}{c}\text { Elongation at } \\
\text { break/\% }\end{array}$ \\
\hline GE-R & $47.40 \pm 0.89$ & $32.66 \pm 0.19$ & $3.89 \pm 0.88$ \\
SSM141 & $47.60 \pm 1.58$ & $60.36 \pm 1.51$ & $29.6 \pm 4.67$ \\
SSM222 & $49.20 \pm 1.22$ & $95.57 \pm 4.44$ & $99.22 \pm 6.05$ \\
PU-Ca & $48.40 \pm 1.58$ & $98.67 \pm 0.40$ & $140.60 \pm 4.57$
\end{tabular}

assays. Specifically, a piece of nanofibrous mat with the size of $1.5^{*} 1.5 \mathrm{~cm}^{2}$ was immersed in $4 \mathrm{~mL}$ DPPH ethanol solution $(1.5$ $\mathrm{mg} / \mathrm{mL}$ ), scavenging activity assay was carried out by recording the absorbance at $516 \mathrm{~nm}$ of DPPH solution at six specific time points, including $2,4,8,16,32$, and 64 min after the mixture reaction, and the lower average absorbance value indicates better antioxidant activity.

\section{RESULTS AND DISCUSSION}

\section{Morphological Analysis}

SEM images of surface and cross-section of electrospun nanofibrous membranes are presented in Figure 1. Firstly, all the as-fabricated nanofibrous mats show desirable fiber morphology and size homogeneity without beads (Figures 1A-D), while slow evaporation of DMAc, bending deformation, entanglement of the nanofibers and resulted in the compacted homogenous structure (Figure 1B), and the average diameter of GE-R and PU-Ca are 127 and $131 \mathrm{~nm}$, respectively. Secondly, the obvious sandwich structure can be observed, and the distinct boundary exists between the adjacent two layers, while interconnected porous structure due to the entanglement of nanofibers are visible from the cross-sectional sites (Figures 1E,F). Unlike the conventional bilayered nanofibrous mats, such kind of sandwich structure can ensure the integration of nanofibrous membrane during the application, in other words, under the protection of bottom and top layers which are hydrophobic and materials-homogenous, such SSM can prevent the mutual stripping, collapse and effusion under the circumstance of water erosion or others. Additionally, the highly porous structure is highly beneficial to the circulation of oxygen gas and water vapor. More importantly, the wound exudates can freely access to the inner hydrogel nanofibrous layer through the porous pathway and stay inside the layer up to the saturated absorption.

\section{FT-IR Characterization and Thermal Property Investigation}

As shown in Figure 2, the typical strong peaks of relevant bonds indicate the blending and electrospinning process did
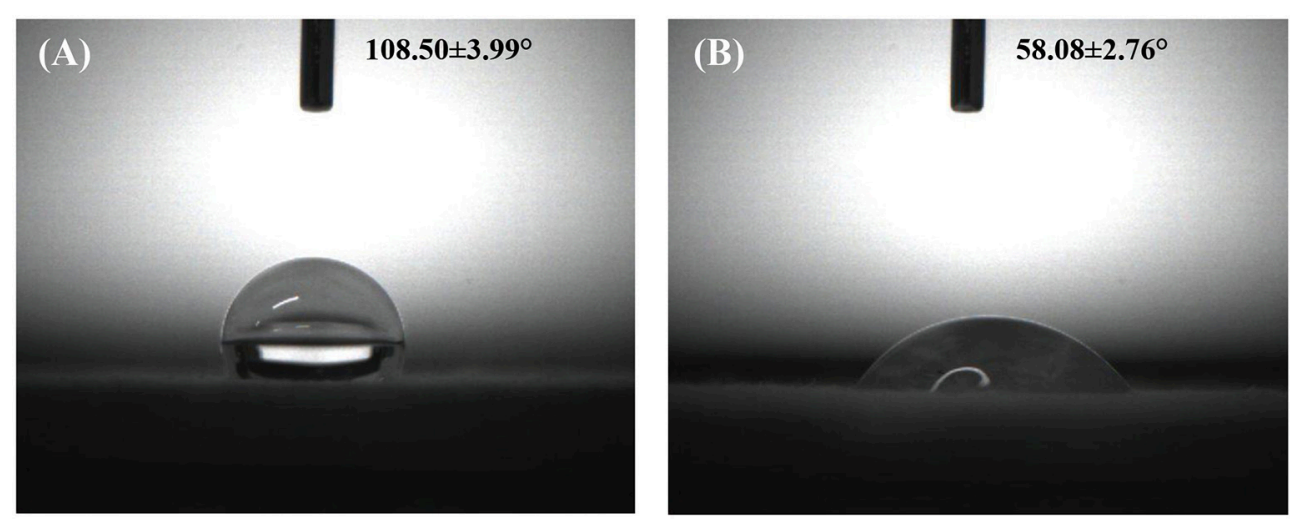

FIGURE 5 | Water contact angle images of different membranes PU-Ca (A) and GE-R (B). 


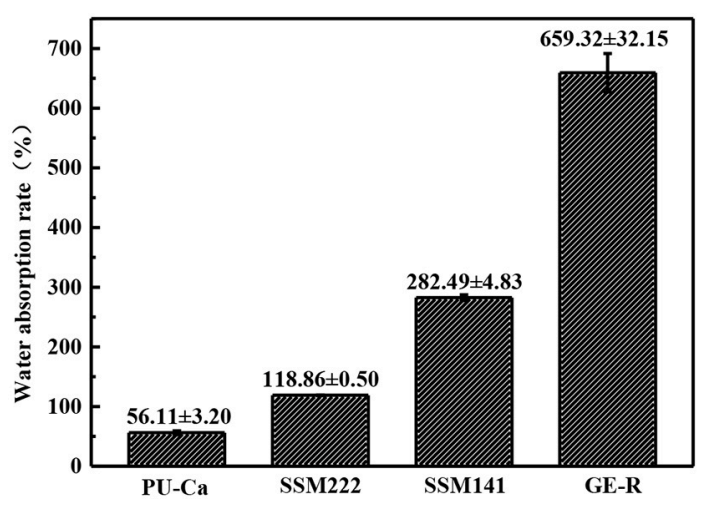

FIGURE 6 | WAR of different electrospun membranes under ambient condition.

not influence the structure of each component. Particularly, it can be clearly observed that the characteristic peak at $\sim 1,642$ $\mathrm{cm}^{-1}$ represents the $\mathrm{C}=\mathrm{O}$ stretching vibration of peptide bonds in the backbone of gelatin, and the peak at $\sim 1,536 \mathrm{~cm}^{-1}$ corresponds to the $\mathrm{N}-\mathrm{H}$ bending and $\mathrm{C}-\mathrm{N}$ stretching vibration; Additionally, the sharp peaks at $\sim 1,722 \mathrm{~cm}^{-1}$ and broad peak at $2,942 \mathrm{~cm}^{-1}$ can be ascribed to $\mathrm{C}=\mathrm{O}$ stretching vibration of urethane groups and stretching vibration of $-\mathrm{NH}$ and $\mathrm{OH}$ groups of PU, respectively (Tan et al., 2015b). After they blended with rutin or antibacterial agent $\mathrm{Ca}$, and even after the formation of SSM, the above typical peaks did not shift, thus, it can be concluded that all the components maintain their own native structure after the physical blending and electrospinning.

The thermal decomposition behavior was also studied, and the decomposition curves are shown in Figure 3, Figures S2A,B. Regarding the semi-decomposition temperature (SDT) and maximum decomposition temperatures (MDT), $167,374,396^{\circ} \mathrm{C}$ and $181,382,408^{\circ} \mathrm{C}$ are recorded for $\mathrm{Ca}, \mathrm{PU}-\mathrm{Ca}$, and $\mathrm{PU}$, that means the thermal stability of PU decreases over $20^{\circ} \mathrm{C}$ after blending with $\mathrm{Ca}$; In contrast, the stability of gelatin was significantly improved after blending with rutin in terms of SDT and MDT, e.g. the MDT of GE-R reach to $326^{\circ} \mathrm{C}$, while only $276^{\circ} \mathrm{C}$ was recorded for the MDT of gelatin (Figures S2C,D). Additionally, from Figures $\mathbf{3 A}, \mathbf{B}$, both SSMs shows desirable thermal stability with near $400^{\circ} \mathrm{C}$ of SDT and above $400^{\circ} \mathrm{C}$ of MDT. In addition, there is no obvious difference between SSM141 and SSM222 in terms of SDT and MDT, indicating that the change of the thickness ratio of each layer has little influence on the thermal stability of the sandwich structural nanofibrous membranes, and such desirable thermal stability can endow the possible thermal sterilization of SSM before use.

\section{Mechanical Properties}

A certain mechanical strength can cater to the motorial behavior of skin, while weak mechanical strength may cause the collapse of wound dressings and then fail to protect the wound bed. Figure 4 shows the representative stressstrain curves of different electrospun membranes. In this study, breaking force and elongation were recorded to evaluate the mechanical properties, and the data were summarized in Table 1. Obviously, GE-R has the lowest breaking force $(\sim 33 \mathrm{cN})$ as well as the breaking elongation $(\sim 4.0 \%)$, thus gelatin in combination with other polymers, e.g., PU, for wound dressing application is preferable. From the testing results, SSMs show much better mechanical property compared with that of GE-R electrospun membrane, the reason can be mainly attributed to the presence of PU-Ca layers, and higher composition ratio of PU-Ca endows SSM better mechanical property, i.e., SSM222 have desirable breaking force of around $96 \mathrm{cN}$ and elongation of around 99\%. Thus, the obtained SSM can withstand the mechanical deformation resulted from the motorial behavior.

\section{Surface Wettability}

Surface wettability is an important property for wound dressing which can influence not only the biological response but the defense performance against water erosion. Figure 5 shows the WCA values of two individual layer surfaces which consist the final SSMs, and the layer of PU-Ca is hydrophobic with a WCA value of $108.50 \pm 3.99^{\circ}$, while the hydrogel nanofibrous layer of GE-R exhibits relative hydrophilic with a WCA value of $58.08 \pm 0.76^{\circ}$. The WCA values of SSM141 and SSM222 were shown in Figure S3, and they have little difference with pristine PU-Ca layer, indicating that the inner layer of the hydrogel nanofiber has no significant effect on the surface wettability of SSM. Consequently, the bottom and top layers of the fabricated SSMs can effectively defense the water or wound fluids erosion and keep the structural integrity of SSMs during the application, and the inner hydrogel nanofibrous layer can absorb the wound fluids which can freely access through the nanofibrous pathway.

\section{WAR and WVTR Study}

Both WAR and WVTR are critical factors for the wound management, and both of them play their own role separately and synergistically. Particularly, WAR is applied to evaluate the exudates retaining capacity of the wound dressing, and WVTR will influence the wounds dehydration or accumulation of exudates. In virtue of desirable WAR and WVTR, an ideal moisture microenvironment will exist around the site of wound bed, thus promote the wound healing process.

WAR and WVTR are calculated according to following Equation (1) and Equation (2). In Equation (1), $\mathrm{W}_{1}$ represents the weight after the suction, and $\mathrm{W}_{0}$ represents the original weight of the sample.

$$
\mathrm{WAR}(\%)=\frac{\mathrm{W}_{1}-\mathrm{W}_{0}}{\mathrm{~W}_{0}} \times 100 \%
$$

$$
\begin{aligned}
& \text { WVTR }\left(\mathrm{mg} . \mathrm{cm}^{-2} \cdot \mathrm{h}^{-1}\right)= \\
& \frac{\text { Water weight change }(\mathrm{mg})}{\text { Exposure area }\left(\mathrm{cm}^{2}\right) \times \text { exposure time }(\mathrm{h})}
\end{aligned}
$$



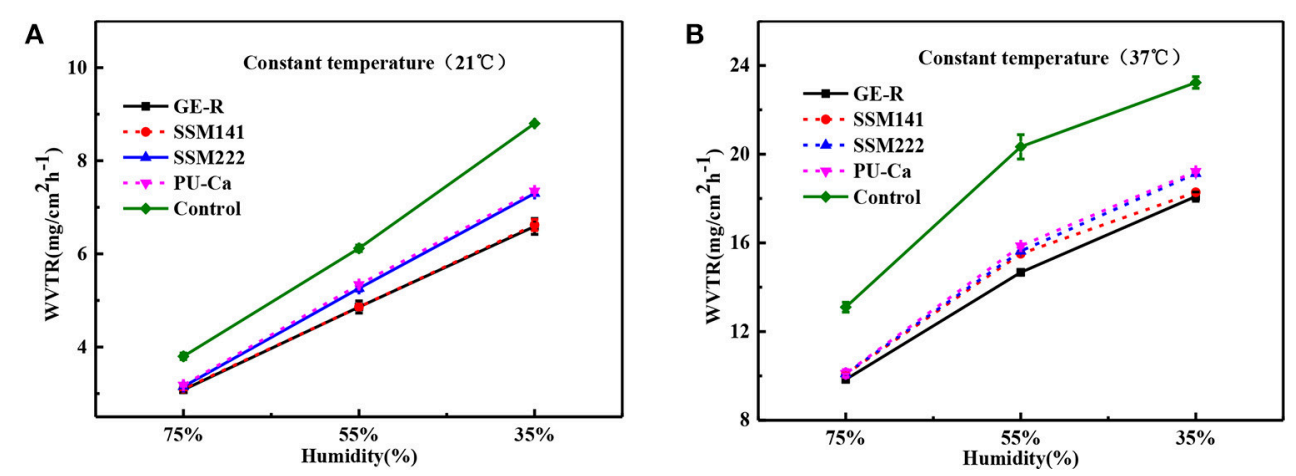

FIGURE 7 | WTR curves by the function of humidity under constant temperature $21^{\circ} \mathrm{C}(\mathbf{A})$ and constant temperature $37^{\circ} \mathrm{C}(\mathbf{B})$.

TABLE 2 | WVTR and WAR of different electrospun membranes.

\begin{tabular}{|c|c|c|c|c|c|c|c|c|}
\hline \multirow[t]{2}{*}{ Samples } & \multirow[t]{2}{*}{ Thickness $(\mu \mathrm{m})$} & \multicolumn{3}{|c|}{$\begin{array}{c}\text { WVTR } / 21^{\circ} \mathrm{C}\left(\mathrm{mg} / \mathrm{cm}^{2} \cdot \mathrm{h}^{-1}\right) \\
\text { Humidity(\%) }\end{array}$} & \multicolumn{3}{|c|}{$\begin{array}{c}\text { WVTR } / 37^{\circ} \mathrm{C}\left(\mathrm{mg} / \mathrm{cm}^{2} \cdot \mathrm{h}^{-1}\right) \\
\text { Humidity(\%) }\end{array}$} & \multirow[t]{2}{*}{ WAR(\%) } \\
\hline & & $35 \%$ & $55 \%$ & $75 \%$ & $35 \%$ & $55 \%$ & $75 \%$ & \\
\hline GE-R & $47.40 \pm 0.89$ & $6.59 \pm 0.17$ & $4.86 \pm 0.13$ & $3.08 \pm 0.03$ & $18.09 \pm 0.21$ & $14.67 \pm 0.02$ & $9.84 \pm 0.07$ & $659.32 \pm 32.15$ \\
\hline SSM141 & $47.60 \pm 1.58$ & $6.62 \pm 0.12$ & $4.86 \pm 0.05$ & $3.10 \pm 0.02$ & $18.28 \pm 0.09$ & $15.51 \pm 0.09$ & $10.08 \pm 0.09$ & $282.49 \pm 2.83$ \\
\hline SSM222 & $49.20 \pm 1.22$ & $7.30 \pm 0.005$ & $5.26 \pm 0.06$ & $3.15 \pm 0.03$ & $19.13 \pm 0.04$ & $15.63 \pm 0.04$ & $10.10 \pm 0.15$ & 118.8600 .50 \\
\hline PU-Ca & $48.60 \pm 1.58$ & $7.35 \pm 0.03$ & $5.34 \pm 0.01$ & $3.18 \pm 0.02$ & $19.22 \pm 0.03$ & $15.86 \pm 0.04$ & $10.11 \pm 0.17$ & $56.11 \pm 3.20$ \\
\hline Control & NA & $8.80 \pm 0.16$ & $6.12 \pm 0.07$ & $3.8 \pm 0.07$ & $23.23 \pm 0.26$ & $20.33 \pm 0.55$ & $13.10 \pm 0.22$ & NA \\
\hline
\end{tabular}
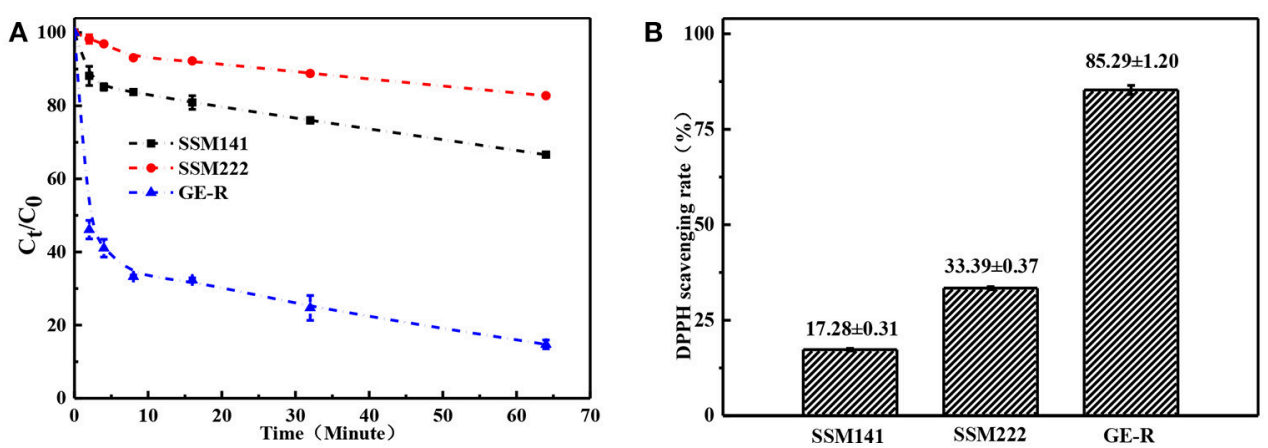

FIGURE 8 | Antioxidant performance of different electrospun membranes. (A) Scavenging rate-time curve of different membranes; (B) Scavenging rate histogram of different membranes.

The thickness of the obtained nanofibrous membranes are around $50 \mu \mathrm{m}$. As shown in Figure 6, the WAR values of PU$\mathrm{Ca}, \mathrm{SSM} 222$, SSM141, and GE-R are around 56, 119, 282, and $659 \%$, respectively. The WAR of SSM222 and SSM 141 increased by 2 and 5 times higher than that of PU-Ca membrane, which indicates the incorporation of hydrogel nanofibrous mat can significantly increase the WAR, and the thicker of GE$\mathrm{R}$ layer incorporated in such sandwich structural membrane renders higher WAR owning to the intrinsic hygroscopicity of gelatin. Therefore, the accumulation of exudates around the wound beds can be avoided and the risk of potential bacteria infection will be reduced. Whatever normal skin or injured skin, body fluid will evaporate through the micropores of skin, while the injured skin normally shows higher WVTR of 1.16$21.41 \mathrm{mg} . \mathrm{cm}^{-2} \cdot \mathrm{h}^{-1}$ compared to that of normal skin (Saeed et al., 2017). Thus, porous structure with suitable WVTR of wound dressing is essential and required. Figure 7 and Figure S4 show the successive recorded WVTR values of different electrospun membranes under different temperature and humidity. It can be clearly seen that the WVTR values for all electrospun membranes reasonably increase with temperature increasing under constant humidity, while WVTR decreases with increasing the ambient humidity. From Table 2, all WVTR values of SSMs, lowest value of $3.10 \pm 0.02 \mathrm{mg} / \mathrm{cm}^{2} \cdot \mathrm{h}^{-1}$ and the highest value of $19.13 \pm$ $0.04 \mathrm{mg} / \mathrm{cm}^{2} \cdot \mathrm{h}^{-1}$, are within the body fluids evaporation range of injured skin, which can indicate that the fabricated SSM can meet 


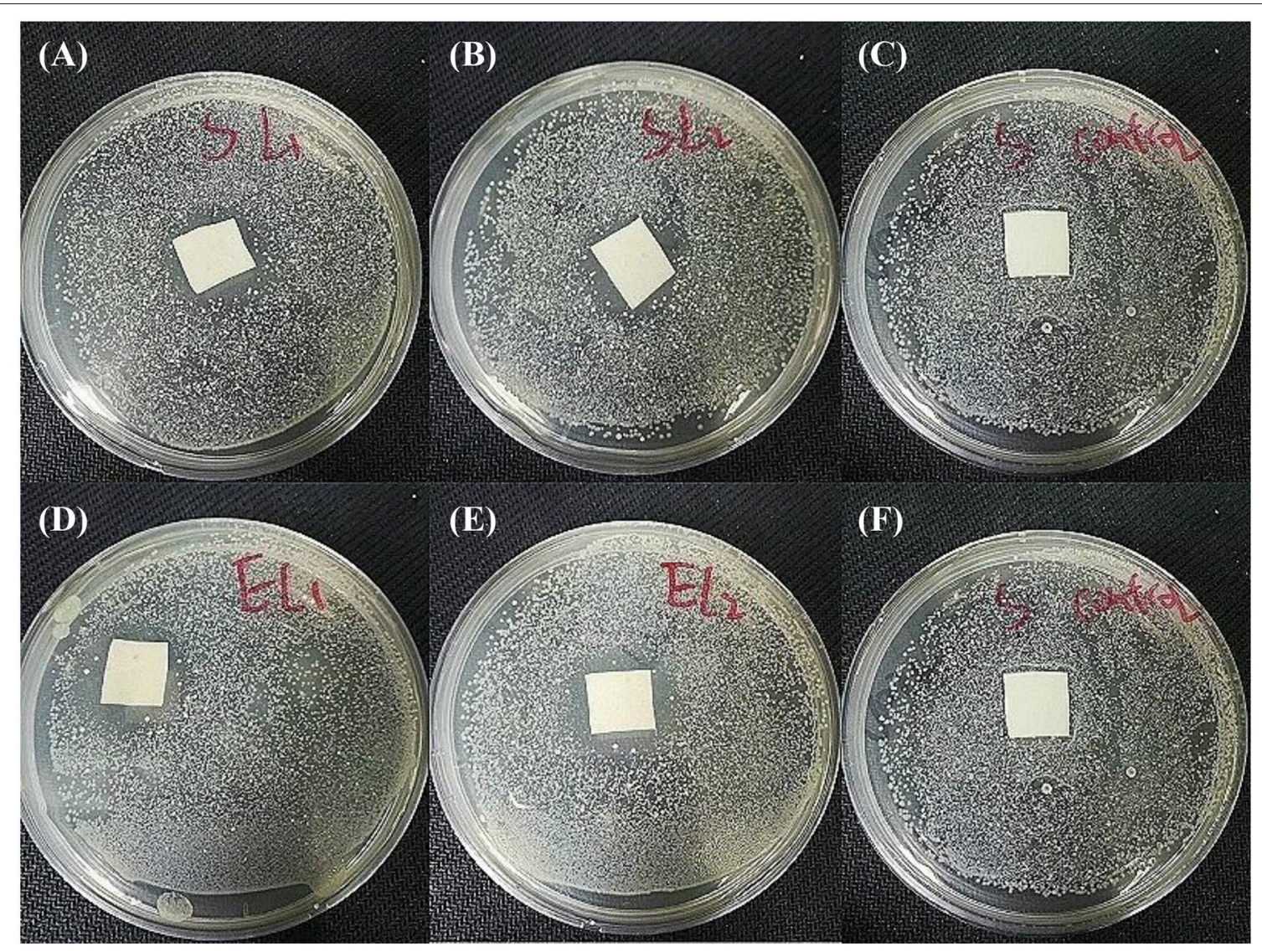

FIGURE 9 | Antibacterial activity investigation results. SSM141against S. aureus (A) and E. coli (D). SSM222 against S. aureus (B) and E. coli (E), and pristine PU against $S$. aureus (C) and E. coli $\mathbf{( F )}$ as the comparisons.

the requirement on managing the wound fluids as a qualified wound dressing.

\section{Antioxidant Property}

Antioxidant property of the fabricated sandwich structural membrane was evaluated in term of the capacity on scavenging free radicals, and the scavenging ratio of DPPH free radicals (SRDPPH) was calculated according to the following Equation (3):

$$
\operatorname{SRDPPH}(\%)=\frac{\mathrm{A}_{0}-\mathrm{A}_{\mathrm{t}}}{\mathrm{A}_{0}} \times 100 \%
$$

Where $A_{0}$ is the original absorbance of DPPH, $A_{t}$ is the absorbance of the DPPH at the specific measuring time. Antioxidant property refers to the ability of scavenging free radicals, and it should be a crucial property for wound dressing which can protect human skin or wound bed from free radicals damage. Antioxidant performance was monitored through measuring the absorbance of DPPH-contained solution at $516 \mathrm{~nm}$, which was also accompanied by a color change from deep purple to yellow. Figure $\mathbf{8}$ demonstrates the antioxidant activity of SSM prepared with different contents of rutin, and higher content of rutin renders the higher SRDPPH, that means higher content of GE-R layer can be incorporated to prepare SSM with better antioxidant performance. In addition, the SRDPPH was recorded by the function of time (Figure $8 B$ ), and the slope of the curve can reflect the scavenging rate of $\mathrm{DPPH}$ free radicals. We can clearly observe that the oxidant performance of all the samples include one initial fast SRDPPH stage with a sharp slope and one slower stage. In other words, the electrospun membranes can initially scavenge the DPPH free radicals quickly then reach a plateau gradually.

\section{Antibacterial Activity}

Antibacterial activity is essential to a qualified wound dressing, that's because a longer healing process will be required once the wound bed is infected by bacteria. Figure 9 shows the antibacterial activity of two SSMs against E. coli and $S$. aureus. Compared with the pristine PU membrane, PU-Ca (Figure S5), SSM141 and SSM222 have satisfactory antibacterial activity by the evidence of obvious inhibition zones. In addition, we observed that the inhibition zones against E. coli is slightly larger than those against $S$. aureus, the possible reason can be attributed to the thicker cell wall of the Gram-positive negative bacteria, and thus the encapsulated antibacterial monomer 
N-halamine (Ca) can more effectively kill the Gram-negative bacteria.

\section{CONCLUSION}

In this study, we have successfully fabricated a novel type of electrospun SSM with a hydrogel nanofibrous mat as the inner layer through lay-by-layer deposition. The obtained SSM has two hydrophobic surfaces (PU-Ca) which can prevent the water erosion, and simultaneously has a high water-uptake capacity because of the hydrophilic hydrogel nanofibrous layer (GE-R), thus the structural integrity can be effectively maintained during the application. Additionally, the SSM also displays favorable antibacterial activity against $E$. coli and $S$. aureus, antioxidant activity in terms of scavenging capacity of DPPH free radicals, and a desirable thermally regulated WVTR. More importantly, the functions and properties can be highly adjusted owing to the nanofibrous sandwich structure, and the incorporated functions can be also performed individually, such as the thickness of inner hydrogel nanofibrous layer, wettability of each layer, mechanical strength and antioxidant period. We believe that such type of SSM can work competently as a wound dressing, and more functional SSMs can be prepared based on this concept.

\section{REFERENCES}

Ahmed, F. E., Lalia, B. S., and Hashaikeh, R. (2015). A review on electrospinning for membrane fabrication: challenges and applications. Desalination 356, 15-30. doi: 10.1016/j.desal.2014.09

Bhowmick, S., and Koul, V. (2016). Assessment of PVA/silver nanocomposite hydrogel patch as antimicrobial dressing scaffold: synthesis, characterization and biological evaluation. Mater. Sci. Eng. C 59, 109-119. doi: 10.1016/j.msec.2015.10.003

Brunelle, A. R., Horner, C. B., and Nam, J. (2017). Electrospun thermosensitive hydrogel scaffold for enhanced chondrogenesis of human mesenchymal stem cells. Acta Biomater. 66, 166-176. doi: 10.1016/j.actbio.2017.11.020

Chen, G., Chen, J., Yang, B., Li, L., Luo, X., Zhang, X., et al. (2015). Combination of aligned PLGA/Gelatin electrospun sheets, native dental pulp extracellular matrix and treated dentin matrix as substrates for tooth root regeneration. Biomaterials 52, 56-70. doi: 10.1016/j.biomaterials.2015.02.011

Dong, A., Wang, Y. J., Gao, Y., Gao, T., and Gao, G. (2017). Chemical insights into antibacterial N-Halamines. Chem. Rev. 117, 4806-4862. doi: 10.1021/acs.chemrev.6b00687

Fan, Z., Liu, B., Wang, J., Zhang, S., Lin, Q., Gong, P., et al. (2014). A novel wound dressing based on $\mathrm{Ag} / \mathrm{Graphene}$ polymer hydrogel: effectively kill bacteria and accelerate wound healing. Adv. Funct. Mater. 24, 3933-3943. doi: 10.1002/adfm.201304202

Feng, Q., Wei, K., Lin, S., Xu, Z., Sun, Y., Shi, P., et al. (2016). Mechanically resilient, injectable, and bioadhesive supramolecular gelatin hydrogels crosslinked by weak host-guest interactions assist cell infiltration and in situ tissue regeneration. Biomaterials 101, 217-228. doi: 10.1016/j.biomaterials.2016.05.043

Gonzalez, J. S., Ludueña, L. N., Ponce, A., and Alvarez, V. A. (2014). Poly(vinyl alcohol)/cellulose nanowhiskers nanocomposite hydrogels for potential wound dressings. Mater. Sci. Eng. C Mater. Biol. Appl. 34, 54-61. doi: 10.1016/j.msec.2013.10.006

Janik, H., and Marzec, M. (2015). A review: fabrication of porous polyurethane scaffolds. Mater. Sci. Eng. C 48, 586-591. doi: 10.1016/j.msec.2014.12.037

Kim, J. W., Kim, M. J., Ki, C. S., Kim, H. J., and Park, Y. H. (2017). Fabrication of bi-layer scaffold of keratin nanofiber and gelatin-methacrylate hydrogel:

\section{AUTHOR CONTRIBUTIONS}

$\mathrm{XY}$, YW, and YL completed the experiments. PL and ZL provided some assistance on conducting experiments and testing. YS, JL, and RG gave some suggestions on writing. LT proposed the idea, designed the experiment, gave scientific support, revised, and finalized the manuscript.

\section{FUNDING}

This work was sponsored by the Fundamental Research Funds for the Central Universities (Grant No. YJ201726), Key Research Program of Science \& Technology Department of Sichuan Province (Grant No. 2017SZYZF00009), Strategic Project of Lu Zhou Science \& Technology Bureau of Sichuan Province (Grant No. 2017CDLZ-S01).

\section{SUPPLEMENTARY MATERIAL}

The Supplementary Material for this article can be found online at: https://www.frontiersin.org/articles/10.3389/fchem. 2018.00490/full\#supplementary-material

implications for skin graft. Int. J. Biol. Macromol. 105(Pt 1), 541-548. doi: 10.1016/j.ijbiomac.2017.07.067

Kucinska-Lipka, J., Gubanska, I., Janik, H., and Sienkiewicz, M. (2015). Fabrication of polyurethane and polyurethane based composite fibres by the electrospinning technique for soft tissue engineering of cardiovascular system. Mater. Sci. Eng. C Mater. Biol. Appl. 46, 166-176. doi: 10.1016/j.msec.2014.10.027

Kurniawan, A., Gunawan, F., Nugraha, A. T., Ismadji, S., and Wang, M. J. (2016). Biocompatibility and drug release behavior of curcumin conjugated gold nanoparticles from aminosilane-functionalized poly(N-vinyl2-pyrrolidone) electrospun nanofibrous mats. Int. J. Pharm. 516, 158-169. doi: 10.1016/j.ijpharm.2016.10.067

Li, H., Wang, M., Williams, G. R., Wu, J., Sun, X., Lv, Y., et al. (2016). Electrospun gelatin nanofibers loaded with vitamins $\mathrm{A}$ and $\mathrm{E}$ as antibacterial wound dressing materials. RSC Adv. 6, 50267-50277. doi: 10.1039/c6ra05092a

Lin, J., Wang, X., Ding, B., Yu, J., Sun, G., and Wang, M. (2012). Biomimicry via Electrospinning. Crit. Rev. Solid State Mater. Sci. 37, 94-114. doi: 10.1080/10408436.2011.627096

Nian, L., Cao, A., Wang, J., Tian, H., Liu, Y., Gong, L., et al. (2018). Viscoelastic and functional properties of Cod-bone gelatin in the presence of xylitol and stevioside. Front. Chem. 6:111. doi: 10.3389/fchem.2018.00111

Oh, S. H., An, D. B., Kim, T. H., and Lee, J. H. (2016). Wide-range stiffness gradient PVA/HA hydrogel to investigate stem cell differentiation behavior. Acta Biomater. 35, 23-31. doi: 10.1016/j.actbio.2016.02.016

Ravichandran, R. K., Sundaramurthi, D., Gandhi, S., Sethuraman, S., and Krishnan, U. M. (2014). Bioinspired hybrid mesoporous silica-gelatin sandwich construct for bone tissue engineering. Microporous Mesoporous Mater. 187, 53-62. doi: 10.1016/j.micromeso.2013.12.018

Resmi, R., Unnikrishnan, S., Krishnan, L. K., and Kalliyana Krishnan, V. (2016) Synthesis and characterization of silver nanoparticle incorporated gelatinhydroxypropyl methacrylate hydrogels for wound dressing applications. J. Appl. Polym. Sci. 134, 1-9. doi: 10.1002/app.44529

Saeed, S. M., Mirzadeh, H., Zandi, M., and Barzin, J. (2017). Designing and fabrication of curcumin loaded PCL/PVA multi-layer nanofibrous electrospun structures as active wound dressing. Prog. Biomater. 6, 39-48. doi: 10.1007/s40204-017-0062-1 
Sahraro, M., Yeganeh, H., and Sorayya, M. (2016). Guanidine hydrochloride embedded polyurethanes as antimicrobial and absorptive wound dressing membranes with promising cytocompatibility. Mater. Sci.Eng. C Mater. Biol. Appl. 59, 1025-1037. doi: 10.1016/j.msec.2015.11.038

Sun, X., Lang, Q., Zhang, H., Cheng, L., Zhang, Y., Pan, G., et al. (2017). Electrospun photocrosslinkable hydrogel fibrous scaffolds for rapid in vivo vascularized skin flap regeneration. Adv. Funct. Mater. 27:1604617. doi: 10.1002/adfm.201604617

Tan, L., Gan, L., Hu, J., Zhu, Y., and Han, J. (2015a). Functional shape memory composite nanofibers with graphene oxide filler. Composites Part A 76, 115-123. doi: 10.1016/j.compositesa.2015.04.015

Tan, L., Hu, J., Huang, H., Han, J., and Hu, H. (2015b). Study of multi-functional electrospun composite nanofibrous mats for smart wound healing. Int. J. Biol. Macromol. 79, 469-476. doi: 10.1016/j.ijbiomac.2015.05.014

Tan, L., Hu, J., and Zhao, H. (2015c). Design of bilayered nanofibrous mats for wound dressing using an electrospinning technique. Mater. Lett. 156, 46-49. doi: 10.1016/j.matlet.2015.04.119

Trinca, R. B., Westin, C. B., Silva, J. A. F. D., and Moraes, M. (2017). Electrospun multilayer chitosan scaffolds as potential wound dressings for skin lesions. Eur. Polym. J. 88, 161-170. doi: 10.1016/j.eurpolymj.2017.01.021

Unnithan, A. R., Sasikala, A. R., Murugesan, P., Gurusamy, M., Wu, D., Park, C. H., et al. (2015). Electrospun polyurethane-dextran nanofiber mats loaded with Estradiol for post-menopausal wound dressing. Int. J. Biol. Macromol. 77, 1-8. doi: 10.1016/j.ijbiomac.2015.02.044

Valizadeh, A., and Mussa, F. S. (2014). Electrospinning and electrospun nanofibres. Iet Nanobiotechnol. 8, 83-92. doi: 10.1049/iet-nbt.2012.0040

Wang, L., Wu, Y., Guo, B., and Ma, P. X. (2015). Nanofiber Yarn/Hydrogel core-shell scaffolds mimicking native skeletal muscle tissue for guiding $3 \mathrm{~d}$ myoblast alignment, elongation, and differentiation. Acs Nano 9, 9167-9179. doi: 10.1021/acsnano.5b03644

Wang, M., Li, X., Hua, W., Shen, L., Yu, X., and Wang, X. (2016). Electrospun Poly(acrylic acid)/Silica hydrogel nanofibers scaffold for highly efficient adsorption of Lanthanide ions and its photoluminescence performance. ACS Appl. Mater. Interf. 8, 23995-24007. doi: 10.1021/acsami.6b08294

Wen, J. Z., Ning, A., Jian, H. Y., Zhou, J., and Yong, M. C. (2015). Tough Al-alginate/Poly(N-isopropylacrylamide) hydrogel with tunable lcst for soft robotics. ACS Appl. Mater. Interf. 7, 1758-1764. doi: 10.1021/am507339r

Wu, Y., Wang, L., Guo, B., and Ma, P. X. (2017). Interwoven aligned conductive nanofiber Yarn/Hydrogel composite scaffolds for engineered 3d cardiac anisotropy. ACS Nano 11, 5646-5659. doi: 10.1021/acsnano. $7 \mathrm{~b} 01062$

Xia, Q., Liu, Z., Wang, C., Zhang, Z., Xu, S., and Han, C. C. (2015). A biodegradable trilayered barrier membrane composed of sponge and electrospun layers: hemostasis and antiadhesion. Biomacromolecules 16, 3083-3092. doi: 10.1021/acs.biomac.5b01099

Xu, R., Luo, G., Xia, H., He, W., Zhao, J., Liu, B., et al. (2015). Novel bilayer wound dressing composed of silicone rubber with particular micropores enhanced wound re-epithelialization and contraction. Biomaterials 40, 1-11. doi: 10.1016/j.biomaterials.2014.10.077

Yao, C., Liu, Z., Yang, C., Wang, W., Ju, X. J., Xie, R., et al. (2015). Poly(Nisopropylacrylamide)-Clay nanocomposite hydrogels with responsive bending property as temperature-controlled manipulators. Adv. Funct. Mater. 25, 2980-2991. doi: 10.1002/adfm.201500420

Zhao, X., Guo, B., Wu, H., Liang, Y., and Ma, P. X. (2018). Injectable antibacterial conductive nanocomposite cryogels with rapid shape recovery for noncompressible hemorrhage and wound healing. Nat. Commun. 9:2784. doi: 10.1038/s41467-018-04998-9

Zhao, X., Li, P., Guo, B., and Ma, P. X. (2015). Antibacterial and conductive injectable hydrogels based on quaternized chitosan-graftpolyaniline/oxidized dextran for tissue engineering. Acta Biomater. 26, 236-248. doi: 10.1016/j.actbio.2015.08.006

Zhao, X., Wu, H., Guo, B., Dong, R., Qiu, Y., and Ma, P. X. (2017) Antibacterial anti-oxidant electroactive injectable hydrogel as selfhealing wound dressing with hemostasis and adhesiveness for cutaneous wound healing. Biomaterials 122, 34-47. doi: 10.1016/j.biomaterials.2017. 01.011

Conflict of Interest Statement: The authors declare that the research was conducted in the absence of any commercial or financial relationships that could be construed as a potential conflict of interest.

Copyright (C) 2018 Yin, Wen, Li, Liu, Li, Shi, Lan, Guo and Tan. This is an open-access article distributed under the terms of the Creative Commons Attribution License (CC $B Y)$. The use, distribution or reproduction in other forums is permitted, provided the original author(s) and the copyright owner(s) are credited and that the original publication in this journal is cited, in accordance with accepted academic practice. No use, distribution or reproduction is permitted which does not comply with these terms. 